\title{
Verbal Fluency Predicts Mortality in Alzheimer Disease
}

\author{
Stephanie Cosentino, PhD, ${ }^{*} \dagger$ Nikolaos Scarmeas, MD, ${ }^{*} \dagger+$ Steven M. Albert, PhD, ${ }^{*} \dagger \neq$ \\ and Yaakov Stern, PhD* $\dagger+$
}

Objectives: To assess the predictive value of neuropsychologic profiles, at diagnosis, for mortality in incident Alzheimer disease (AD).

Background: Rate of AD progression varies significantly across individuals for reasons that are not well understood. Several studies have linked rapid decline with disproportionately impaired executive functioning, presumably reflecting greater impairment of frontal networks. To the extent that differential neuropsychologic profiles reflect various neuropathologic presentations of AD, such profiles may inform survival estimates early in the disease.

Methods: Five neuropsychologic indices were used to characterize performance in 161 individuals at diagnosis of AD during a 15-year, longitudinal, primarily community-based study.

Results: Fifty-two percent of participants reached the mortality end point with a median survival of 5.52 years $(95 \%$ confidence interval, 4.41-6.63). Cox proportional hazards analyses indicated that older age at diagnosis was associated with higher risk of mortality (risk ratios, 1.08; 95\% confidence interval, 1.04-1.12) whereas Hispanic ethnicity predicted lower mortality [0.22 (0.09-0.55)]. Controlling for these 2 demographic variables, higher verbal fluency scores at diagnosis predicted lower mortality [0.69 (0.49-0.96)].

Conclusions: Disproportionate impairment of both category and letter fluency at the earliest stages of AD predicts mortality. The prognostic value of these tests may derive from their general psychometric properties, or may reflect the measures' sensitivity to an early or critical level of compromise to frontal networks.

Key Words: Alzheimer disease, mortality, verbal fluency, prognosis, neuropsychology

(Cog Behav Neurol 2006;19:123-129)

Received for publication November 18, 2005; accepted March 26, 2006. From the *Cognitive Neuroscience Division of the Taub Institute for Research in Alzheimer's Disease and the Aging Brain; †Gertrude H. Sergievsky Center; and \$Department of Neurology, Columbia University Medical Center, NY.

Supported by federal grants AG0732, AG00261, and RR00645. A portion of this work was presented at the 57th Annual Meeting of the American Academy of Neurology in Miami Beach, FL (April 2005).

Reprints: Yaakov Stern, PhD, Columbia University Medical Center, 630 West 168th Street, P\&S Box 16, NY 10032 (e-mail: ys11@ columbia.edu).

Copyright $(102006$ by Lippincott Williams \& Wilkins
$\mathrm{T}$ he median survival time after a diagnosis of Alzheimer disease (AD) has been estimated at 7 to 10 years in patients diagnosed in their $60 \mathrm{~s}$ and $70 \mathrm{~s}$, and 3 years in patients diagnosed in the eighth or ninth decade of life..$^{1,2}$ However, rate of disease progression varies significantly among individuals. Rapid decline has been linked to early age of onset, male gender, ${ }^{3}$ concurrent physical illness, ${ }^{4}$ coexisting depression, ${ }^{5}$ extrapyramidal symptoms, ${ }^{6}$ gait disturbance and vascular risk factors 7 ; however, the factors which determine individual rate of progression are not well understood, particularly those identifiable early in the course of AD. Marked global cognitive impairment on the Mini-Mental State Examination ${ }^{8}$ seems to predict mortality most consistently ${ }^{4,9}$; however, ceiling effects render this test less useful in early AD when patients achieve relatively high scores. ${ }^{10}$ In contrast, heterogeneous neuropsychologic profiles across individuals with early AD offer the opportunity to evaluate the prognostic value of differential deficits in specific cognitive domains for disease course, or mortality. ${ }^{11-13}$

Although patients with AD exhibit a core memory deficit, there is considerable variability in performance on measures of executive functioning, language, and visuospatial skills, so much so that various "subtypes" of AD including frontal and posterior variants have been described. ${ }^{12,14-17}$ Functional neuroimaging and neuropathologic studies have suggested that distinct neuropsychologic profiles map onto differential distributions of neuropathology reflected through regional hypometabo$\operatorname{lism}^{11,18,19}$ and burden of amyloid plaques on autopsy. ${ }^{20}$ To the extent that various neuropathologic presentations of AD may be related to differential survival rates, analysis of neuropsychologic profiles at diagnosis has the potential to inform prognosis early in the disease course. Preliminary evidence from neuropsychologic ${ }^{19,21}$ and neuroimaging ${ }^{19}$ investigations in patients with prevalent $\mathrm{AD}$ has suggested that early and disproportionate involvement of frontal networks may herald a more rapid disease course. Overall, however, the relationship between neuropsychologic profile and disease course has been explored only minimally, and existing studies have not followed patients from the earliest stages of AD until mortality.

The current study evaluates the usefulness of 5 neuropsychologic indices measured at diagnosis, including memory, abstract reasoning, language, visuospatial functioning, and verbal fluency in predicting differential mortality risk in patients with incident AD. Existing work suggests that skills dependent on the integrity of frontal 
networks, such as abstract reasoning and verbal fluency, may be particularly useful in predicting survival. ${ }^{41-43}$

\section{MATERIALS AND METHODS}

\section{Participants}

For the present analyses, AD patients were identified and followed through 2 cohorts described in more detail in earlier work. ${ }^{22,23}$ Briefly, the first cohort consisted of a community registry of subjects enrolled between 1989 and 1992 from regional medical facilities (inpatient and outpatient services and private practitioners in the community), nursing homes serving local residents, a state agency list of home care recipients, senior centers and housing, volunteers or self-referred, and some spouses of individuals identified as cases. Because of the enrollment procedure of this cohort, it may not be completely representative of the community; however, only 23 subjects in the present analyses were from this cohort. The majority $(n=138)$ of incident AD patients were identified in a later cohort from the Washington Heights and Inwood Columbia Aging Project, enrolled starting 1992. This cohort consists of elders identified from a probability sample of Medicare beneficiaries residing in the 14 census tracts comprising the area of Manhattan between (approximately) 155th and 181st streets. Access to the names of individuals was provided by the Health Care Financing Administration. The proportion of individuals within each ethnic group and age stratum who participated in the study did not differ significantly from the source population.

Participants were selected for the current study if they were diagnosed with incident $\mathrm{AD}$, that is, if they did not meet criteria for dementia upon entry into the study, but converted to a diagnosis of dementia at a follow-up evaluation. Further, as we were interested in examining disease course, we included only those subjects on whom postincident data was available (eg, follow-up cognitive assessment or mortality data). The final analysis included 161 subjects who were diagnosed with incident $A D$, had follow-up data available, and continued to meet criteria for dementia at their last evaluation. Two hundred seventy two nondemented control cases were matched with the incident $\mathrm{AD}$ sample on age, education, and ethnicity, and included in the current study to provide normative data for neuropsychologic measures.

\section{Procedures}

The study cohort was followed over a 15-year period beginning in 1989 during which time each participant received the same medical, neurologic, and neuropsychologic evaluations at approximately 20 -month intervals. A physician elicited each subject's medical and neurologic history and conducted a standardized physical and neurologic examination. All ancillary information (medical charts, computed tomographies or magnetic resonance imagings) was considered in the evaluation, if available. Medical diagnoses were assigned when applicable. This examination was repeated at each follow-up.
Past medical history was recorded with specific attention to stroke, trauma, medications, and recreational drug use. Participants' medical comorbidities were computed using a modified version of the Charlson Index of Comorbidity $^{24}$ that assessed conditions including myocardial infarct, congestive heart failure, peripheral vascular disease, hypertension, chronic obstructive pulmonary disease, arthritis, gastrointestinal disease, mild liver disease, diabetes, chronic renal disease, and systemic malignancy. All items received weights of 1 , with the exception of chronic renal disease and systemic malignancy, which were weighted 2. For the current study, subjects were assigned the maximum Charlson score obtained during their participation in the study to account for significant comorbidities arising at any point before their death.

All participants underwent a standard neuropsychologic battery that tested multiple domains including memory, orientation, abstract reasoning, language, and visuospatial abilities. The test battery included: The Selective Reminding Test (SRT), a serial list learning task comprised of recall and recognition components ${ }^{25}$; the Benton Visual Retention Test (BVRT) forced-choice recognition task in which participants are asked to identify a geometric figure from an array of 4 figures after an immediate delay ${ }^{26}$; orientation items from the modified Mini-Mental State Examination ${ }^{8}$; Boston Naming Test ${ }^{27}$; Controlled Oral Word Association Test (CFL) and Category Naming-Animals, Food, and Clothing ${ }^{28}$; Boston Diagnostic Aphasia Examination (BDAE) Complex Ideational Material and Repetition subtests ${ }^{29}$; Wechsler Adult Intelligence Scales-Revised (WAIS-R) Similarities subtest ${ }^{30}$; Mattis Dementia Rating Scale (DRS)-Identities and Oddities subtest ${ }^{31}$; Rosen Drawing Test ${ }^{32}$; and the BVRT perceptual matching task. ${ }^{26}$ Subjects were tested in English or Spanish according to their preference.

Subjects performing below specified cutoff scores for 2 memory measures, and in 2 other cognitive domains, were considered to have sufficient cognitive impairment to meet cognitive criteria for AD. These cutoff scores, discussed in detail in an earlier paper, ${ }^{33}$ were selected for their usefulness in distinguishing between normal controls and patients with dementia. In addition to impaired neuropsychologic performance, diagnosis of AD required impairment in social or occupational functioning as outlined by the Diagnostic and Statistical Manual of Mental Disorders. ${ }^{34}$ Information from the neurologic, psychiatric, and neuropsychologic assessments was reviewed in a consensus conference comprised of neurologists and neuropsychologists. On the basis of this review, all participants were assigned to 1 of 3 categories: dementia, mild cognitive impairment, or normal cognitive function. Only participants with incident AD were included in this study. That is, they did not meet criteria for dementia upon entry into the study, and later converted to a diagnosis of dementia at a follow-up evaluation. Subjects characterized as having normal cognitive function were included as controls. All 
procedures were approved by the Institutional Review Board at Columbia University Medical Center.

\section{Statistical Analyses}

Cox proportional hazard analysis was used to determine the predictive use of demographic and neuropsychologic variables for mortality. Information regarding mortality, the outcome of interest, was collected through follow-up and the National Death Index. Although age is sometimes used as the duration variable in Cox models, the current study defined duration as time from the AD diagnosis to death or last follow-up, the specific time frame of interest. Given the clear association between age and mortality, however, the predictive value of age was evaluated in the first Cox proportional hazards analysis, along with other potential predictors including gender, education, global cognition, medical comorbidities, and ethnicity. Gender was coded with male as the reference category. Participants were categorized according to ethnicity: Black (African-American, non-Hispanic), White (non-Hispanic), or Hispanic; we used ethnicity as a series of dummy variables with White as the reference category. Years of education, global cognition (average $z$-score of the 5 neuropsychologic indices), and medical comorbidities were entered as continuous variables. Variables that significantly predicted mortality in this model were included as covariates in the remaining Cox proportional hazards analyses.

Five additional Cox models examined the predictive use of each neuropsychologic index measured at diagnosis, entered as a continuous variable predictor for mortality. To create these indices, raw scores on individual tests were converted into standardized $z$-scores on the basis of the means and standard deviations of the matched control group (Table 2). Individual test $z$-scores were then averaged to create a single $z$-score for each index. If fewer than half of the individual $z$-scores were missing for a given domain, the composite score was calculated using the existing data. If greater than half of the tests were missing, the domain score was considered missing data. The 5 neuropsychologic indices were compiled to represent performance in areas including Memory (SRT total recall; SRT delayed recall; BVRT Recognition), Abstract Reasoning (WAIS Similarities; Identities and Oddities), Visuospatial Functioning (Rosen Drawing; BVRT Matching), Language (Boston Naming Test; BDAE Repetition and Complex Ideational Material subtests), and Verbal Fluency (Category Naming and COWA). A follow-up repeated measures analysis of variance was used to evaluate differences on neuropsychologic indices, at diagnosis, across participants who survived and those who died.

\section{RESULTS}

Thirty-nine subjects were lost to follow-up. This group did not differ from the final sample $(n=161)$ in age, education, ethnicity, or scores on 4 of the 5 neuropsychologic indices. However, subjects lost to follow-up had significantly lower medical comorbidities
TABLE 1. Demographic and Clinical Characteristics of Incident AD Group and Nondemented Controls

\begin{tabular}{lcc}
\hline & $\begin{array}{c}\text { Incident AD } \\
(\mathbf{n}=\mathbf{1 6 1})\end{array}$ & $\begin{array}{c}\text { Nondemented } \\
\text { Controls (n= 262) }\end{array}$ \\
\hline Age M (SD) & $82.73(6.72)$ & $80.12(6.15)$ \\
Education M (SD) & $7.10(4.39)$ & $8.24(4.06)$ \\
Comorbidity Index M (SD) & $3.56(1.84)$ & $2.69(1.70)$ \\
Hispanic N (\%) & $89(58 \%)$ & $163(60 \%)$ \\
African American N (\%) & $52(34 \%)$ & $77(28 \%)$ \\
Caucasian N (\%) & $13(8 \%)$ & $32(12 \%)$ \\
Memory & $-1.81(0.45)$ & NA \\
Abstract reasoning & $-0.90(0.85)$ & NA \\
Language & $-0.89(0.92)$ & NA \\
Visuospatial skills & $-0.92(1.10)$ & NA \\
Verbal fluency & $-1.16(0.71)$ & NA
\end{tabular}

Comorbidity Index indicates Modified Charlson Index of Comorbidity.

$(M=2.7, \mathrm{SD}=1.8), t(192)=2.2, P=0.03$, and higher abstract reasoning scores at diagnosis $(M=-0.6$, $\mathrm{SD}=0.7), t(208)=2.2, P=0.03$ than subjects included in the final analysis. The final group of 161 subjects with incident $\mathrm{AD}$ was followed after incidence for an average of 3.90 (2.69) years, with total follow-up times (from diagnosis to last visit or death) ranging from 0.11 to 11.64 years. Fifty-two percent of the incident AD group reached the mortality end point with a median survival of 5.52 years (95\% confidence interval, 4.41-6.63). Table 1 presents the demographic characteristics of the sample. Table 2 presents the normative neuropsychologic data.

In the first Cox model, older age was associated with higher risk of mortality (risk ratios, 1.08; 95\% confidence interval, 1.04-1.12), whereas Hispanic ethnicity predicted lower mortality [0.22 (0.09-0.55)]. Gender was nearly significant, whereas education, global cognition, and medical comorbidities were not significant predictors (Table 3). Controlling for age, Hispanic ethnicity, and gender in the remaining Cox models, higher scores on the verbal fluency index at diagnosis reduced the risk of mortality [0.71 (0.52-0.98); Table 3 and Fig. 1].

Significant variability in scores at the diagnostic visit existed for all neuropsychologic indices with the distribution of $z$-scores shown in Table 4.

A repeated measures analysis of variance with a 2 (survived, deceased) $\times 5$ (memory, language, abstract reasoning, visuospatial functioning, and verbal fluency) design was conducted to evaluate the relative differences in verbal fluency and other neuropsychologic scores, at diagnosis, in patients who died and in those who remained alive. As this analysis excludes subjects who have missing data for any of the neuropsychologic indices, only 120 of 161 subjects were included. A significant main effect was found for scores across the 5 neuropsychologic indices, $F(4,120)=16.97, P=0.000$. As expected, post-hoc $t$ tests revealed that the entire sample performed significantly lower on the memory index than all other indices $(P<0.01)$. Additionally, verbal fluency was significantly lower than the language, 
TABLE 2. Raw Neuropsychologic Scores in Nondemented Normative Sample Matched for Age, Education, and Ethnicity

\begin{tabular}{lcccc}
\hline Test (Maximum Score Possible) & Index & N & Mean & SD \\
\hline SRT total recall (72) & Memory & 266 & 38.95 & 8.57 \\
SRT delayed recall (12) & Memory & 266 & 5.85 & 2.23 \\
BVRT recognition (10) & Memory & 258 & 6.41 & 2.13 \\
WAIS-R similarities (28) & Abstract reasoning & 260 & 8.97 & 6.38 \\
DRS identities and oddities (16) & Abstract reasoning & 253 & 14.26 & 1.63 \\
BNT (15) & Language & 254 & 13.58 & 1.59 \\
BDAE repetition (8) & Language & 257 & 7.57 & 0.87 \\
BDAE comprehension (6) & Language & 257 & 4.98 & 1.20 \\
Rosen (5) & Visuospatial & 254 & 2.37 & 0.96 \\
BVRT matching (10) & Visuospatial & 260 & 8.22 & 1.77 \\
COWAT (N/A) & Verbal fluency & 256 & 8.53 & 3.47 \\
Category Naming (N/A) & Verbal fluency & 259 & 13.40 & 3.41 \\
\hline
\end{tabular}

BDAE indicates Boston Diagnostic Aphasia Examination; BNT, Boston naming Test; BVRT, Benton Visual Recognition Test; COWA, Controlled Oral Word Association Test (CFL); DRS, Dementia Rating Scale; SRT, Selective Reminding Test; WAIS-R, Wechsler Adult Intelligence Scales-Revised.

abstract reasoning, and visuospatial processing indices $(P<0.01)$. Between group analyses demonstrated that memory scores were equally low in both survivors and nonsurvivors, but that the latter group seemed to drive the decreased verbal fluency scores. There was no main effect for group and no significant interaction effect; however, differences across the survivors and nonsurvivors approached significance on the verbal fluency index only $(P=0.06$; Table 5 and Fig. 2$)$.

\section{DISCUSSION}

Early AD is marked by a core amnestic syndrome and heterogeneous deficits in visuospatial functioning, language abilities, and executive skills. ${ }^{11,12,14-16,18,19,35}$ The various cognitive profiles seen in AD are thought to reflect the relative distribution of neuropathology, and have been associated with distinct functional neuroimaging patterns. ${ }^{11,18,19}$ For example, predominantly parietal

TABLE 3. Demographic and Neuropsychologic Predictors of Mortality Incident AD

\begin{tabular}{lccc}
\hline Predictors & Risk Ratios & $\mathbf{9 5 \%}$ CI & $\boldsymbol{P}$ \\
\hline Age & $\mathbf{1 . 0 8}$ & $\mathbf{1 . 0 4 - 1 . 1 2}$ & $\mathbf{0 . 0 0}$ \\
Education & 1.01 & $0.95-1.07$ & 0.72 \\
Gender & 0.57 & $0.32-1.02$ & 0.06 \\
Global cognition & 1.00 & $0.99-1.00$ & 0.70 \\
Comorbidity index & 1.05 & $0.91-1.20$ & 0.52 \\
Hispanic & $\mathbf{0 . 2 4}$ & $\mathbf{0 . 0 9}-\mathbf{0 . 6 2}$ & $\mathbf{0 . 0 0}$ \\
African American & 0.56 & $0.22-1.45$ & 0.23 \\
Memory & 0.95 & $0.58-1.54$ & 0.82 \\
Abstract reasoning & 0.89 & $0.67-1.19$ & 0.44 \\
Visuospatial function & 0.97 & $0.77-1.22$ & 0.79 \\
Language & 1.07 & $0.82-1.40$ & 0.63 \\
Verbal fluency & $\mathbf{0 . 7 1}$ & $\mathbf{0 . 5 2 - 0 . 9 8}$ & $\mathbf{0 . 0 4}$ \\
\hline
\end{tabular}

Cox models for occurrence of death as predicted by demographic and neuropsychologic variables in all subjects. Risk ratios and $95 \%$ confidence intervals $(\mathrm{CI})$ are tabulated, with significant findings in bold $(95 \% \mathrm{CI}$ not including the value 1.0). Risk ratios for ethnicity are reported in reference to white subjects. The 7 demographic variables were entered into 1 Cox model. Neuropsychologic predictors were entered into 5 separate Cox models. or prefrontal hypometabolism on positron emission tomography, has been associated with disproportionately impaired visuospatial and executive skills, respectively. ${ }^{11}$ Collectively, neuropsychologic and neuroimaging data implicate multiple pathways by which AD neuropathology progresses, and reveal the regional disease expression in individual patients. To the extent that different disease pathways are associated with illness duration, early neuropsychologic performance has the potential to inform survival estimates. The current study compared the predictive value of numerous neuropsychologic measures

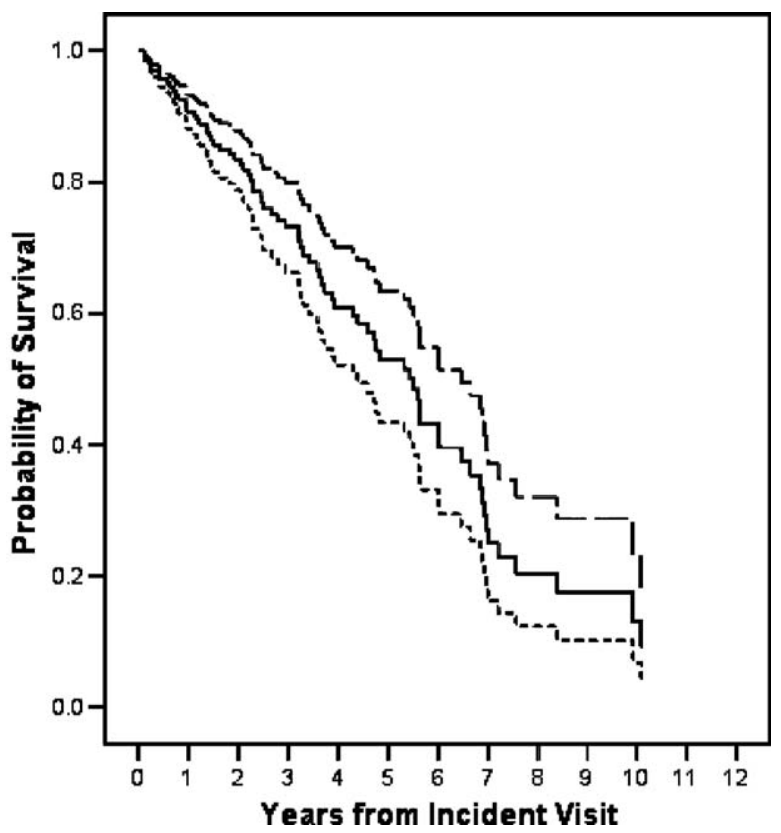

FIGURE 1. Probability of survival in incident $A D$ as a function of verbal fluency at diagnosis. Tertile values are reported in $z-$ scores. Small broken line indicates first tertile (VF $<-1.50)$; solid line, second tertile $(-1.50 \leq \mathrm{VF}<-0.91)$; large broken line, third tertile (VF $\geq-0.91)$. VF indicates Verbal Fluency Index. 
TABLE 4. Distribution of z-scores on Neuropsychologic Indices at Incident Visit

\begin{tabular}{lccccc}
\hline & Memory & Abstract Reasoning & Language & Visuospatial Skills & Verbal Fluency \\
\hline Minimum & -3.2 & -3.2 & -3.3 & -3.3 & -3.6 \\
25th percentile & -2.1 & -1.3 & -1.5 & -1.8 & -1.6 \\
50th percentile & -1.8 & -0.8 & -0.8 & -0.8 & -1.2 \\
75th percentile & -1.5 & -0.2 & -0.2 & -0.2 & -0.7 \\
Maximum & -0.9 & 0.8 & 0.8 & 1.1 & 1.3 \\
\hline
\end{tabular}

for mortality in a multiethnic, primarily communitybased study of incident AD.

The relevance of specific neuropsychologic deficits for rate of $\mathrm{AD}$ progression has been explored only minimally over the past 2 decades, and inconsistent methodology has made it challenging to identify a meaningful relationship between neuropsychologic performance and mortality risk. Although an early study suggested that severe aphasia was the best predictor of mortality, the predictive use of other neuropsychologic deficits was not evaluated. ${ }^{36}$ Further, subjects likely varied in the etiology of their illness as the study's inclusion criteria did not require a memory deficit. A later investigation found that in contrast to memory and language scores, baseline visuoconstructional performance best predicted mortality ${ }^{9}$; however, notably missing in this analysis was assessment of executive abilities.

The current study evaluated the predictive value of a range of neuropsychologic abilities measured at diagnosis in patients with incident AD. The index of verbal fluency was the only significant neuropsychologic predictor of mortality. At diagnosis, each additional unit of performance on the fluency index ( $1 z$-score, equivalent to approximately 3.5 words on each test) was associated with approximately a $30 \%$ risk reduction for reaching the mortality end point. One possible interpretation of these findings is that the prognostic value of verbal fluency derives from its sensitivity to prefrontal, or frontalsubcortical compromise. Both letter and category fluency tasks have substantial executive demands, including systematic search, word retrieval, and working memory subserved in part by prefrontal circuitry. ${ }^{37}$ Theoretically, category fluency draws more heavily than letter fluency upon semantic networks supported by temporal cortex; involvement of this area in early AD generally leads to disproportionate impairment on category rather than letter fluency. ${ }^{35,38,39}$ Accompanying deficits on letter fluency, frequently observed further in the course of the illness, are thought to reflect the extent to which

TABLE 5. Neuropsychologic Scores at Diagnosis by Mortality in Incident AD Participants (Means and Standard Deviations)

\begin{tabular}{lccc}
\hline Indices & Surviving $(\mathbf{n}=\mathbf{5 8})$ & Dead $(\mathbf{n}=\mathbf{6 2})$ & $\boldsymbol{P}$ \\
\hline Memory & $-1.79(0.44)$ & $-1.75(0.43)$ & 0.81 \\
Abstract reasoning & $-0.82(0.85)$ & $-0.90(0.87)$ & 0.50 \\
Visuospatial & $-0.98(1.04)$ & $-0.89(1.15)$ & 0.68 \\
Language & $-0.86(0.96)$ & $-0.76(0.85)$ & 0.53 \\
Verbal fluency & $-0.97(0.72)$ & $-1.27(0.72)$ & 0.06 \\
\hline
\end{tabular}

neuropathology impacts prefrontal cortex. ${ }^{40}$ As such, more severe impairment on both fluency tasks at diagnosis may signal a relatively rapid disease course, or a qualitatively different disease presentation associated with reduced survival.

At least 2 studies have linked rapid disease progression in $\mathrm{AD}$ with early and disproportionate executive dysfunction or prefrontal hypometabolism. ${ }^{19,21}$ Additionally, a recent study linked impaired performance on verbal fluency and digit span backward to reduced survival rates in patients with frontotemporal dementia. ${ }^{41}$ This finding may reflect the fact that patients with the poorest prognosis, those with coexisting motor neuron disease, ${ }^{41,42}$ may have the greatest difficulty on measures of executive functioning; however, it is also possible that independent of motor neuron disease, mortality risk may increase with frontal-subcortical compromise to the extent that corresponding symptoms of apathy eventually contribute to akinetic-mutism, or that aspects of behavioral disturbance lead to risky behaviors or

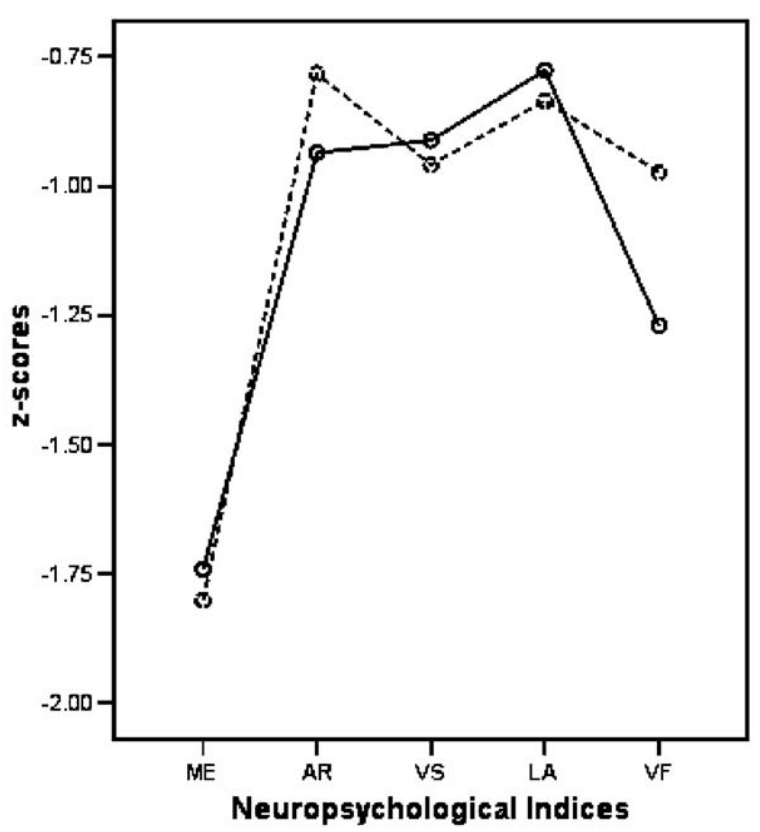

FIGURE 2. Neuropsychologic performance at diagnosis in participants with incident AD. Solid line indicates deceased group; broken line, living group; $M E$, Memory Index; $A R$, Abstract Reasoning Index; VS, Visuospatial Index; LA, Language Index; VF, Verbal Fluency Index. 
overmedication. $^{41}$ Certainly the processes leading to mortality differ across frontotemporal dementia and $\mathrm{AD}$; however, the latter hypothesis may be relevant for the relationship between verbal fluency scores and survival rates in the current study.

Alternatively, the prognostic value of verbal fluency may be primarily a function of its psychometric properties (eg, ample range, lack of floor, or ceiling effects). Verbal fluency and other language scores have demonstrated sensitivity to cognitive impairment across mild-to-moderate $\mathrm{AD}$ in contrast to memory and visuospatial measures which were optimally sensitive at the earlier and later disease stages, respectively. ${ }^{10}$ To the extent that verbal fluency measures are sensitive to cognitive change across the duration of $\mathrm{AD}$, such scores may offer greater prognostic use than other neuropsychologic measures that less closely track disease progression.

One limitation of the current study is that the neuropsychologic battery was originally compiled for diagnostic purposes, and is thus relatively limited in scope. As a result, the selected neuropsychologic indices may not comprehensively capture the stated constructs, potentially preventing us from detecting a true relationship between specific early deficits and mortality. It is also true that certain measures have limited sensitivity owing to ceiling effects in early AD. However, compilation of the diagnostic measures into indices partially addresses this issue, as is evidenced by the wide distribution of scores on the visuospatial, language, and abstract reasoning indices at diagnosis. The variability in scores on each index argues against the possibility that restricted range limited the predictive value of certain indices for mortality.

A second limitation of this study is that the diagnostic algorithm may have constrained our findings (ie, the memory index may not have been predictive of mortality because subjects were required to demonstrate impairment on 2 of 3 memory indices to meet criteria for dementia). However, this is necessitated by the diagnostic criteria for AD. Third, because the participants were not autopsy-confirmed cases of $\mathrm{AD}$, there is a risk that participants with other forms of dementia were included in this study. However, the applied standard diagnostic criteria have a sensitivity of approximately $81 \%$ with a specificity of approximately $70 \% .{ }^{43}$ Further, all diagnoses were subject to physician input at an experienced center, and only those subjects who met criteria for AD at 2 visits were included in the current analyses. Finally, our strict cognitive inclusion criteria may have resulted in the inclusion of slightly more impaired participants.

\section{REFERENCES}

1. Brookmeyer R, Corrada MM, Curriero FC, et al. Survival following a diagnosis of Alzheimer disease. Arch Neurol. 2002;59:1764-1767.

2. Wolfson C, Wolfson DB, Asgharian M, et al. A reevaluation of the duration of survival after the onset of dementia. $N$ Engl J Med. 2001;344:1111-1116.

3. Stern Y, Tang MX, Albert MS, et al. Predicting time to nursing home care and death in individuals with Alzheimer disease. JAMA. 1997;277:806-812.
4. Ueki A, Shinjo H, Shimode H, et al. Factors associated with mortality in patients with early-onset Alzheimer's disease: a five-year longitudinal study. Int J Geriatr Psychiatry. 2001;16:810-815.

5. Burns A, Lewis G, Jacoby R, et al. Factors affecting survival in Alzheimer's disease. Psychol Med. 1991;21:363-370.

6. Stern Y, Albert M, Brandt J, et al. Utility of extrapyramidal signs and psychosis as predictors of cognitive and functional decline, nursing home admission, and death in Alzheimer's disease: prospective analyses from the Predictors Study. Neurology. 1994; 44:2300-2307.

7. Larson EB, Shadlen MF, Wang L, et al. Survival after initial diagnosis of Alzheimer disease. Ann Intern Med. 2004;140:501-509.

8. Folstein MF, Folstein SE, McHugh PR. "Mini-mental state". A practical method for grading the cognitive state of patients for the clinician. J Psychiatr Res. 1975;12:189-198.

9. Hui JS, Wilson RS, Bennett DA, et al. Rate of cognitive decline and mortality in Alzheimer's disease. Neurology. 2003;61:1356-1361.

10. Mungas D, Wallace R, Reed B. Dimensions of cognitive ability in dementia: differential sensitivity to degree of impairment in Alzheimer's disease. Clin Neuropsychologist. 1998;12:129-142.

11. Haxby JV, Grady CL, Koss E, et al. Heterogeneous anteriorposterior metabolic patterns in dementia of the Alzheimer type. Neurology. 1988;38:1853-1863.

12. Galton CJ, Patterson K, Xuereb JH, et al. Atypical and typical presentations of Alzheimer's disease: a clinical, neuropsychological, neuroimaging and pathological study of 13 cases. Brain. 2000;123 (Pt 3):484-498.

13. Fisher NJ, Rourke BP, Bieliauskas LA. Neuropsychological subgroups of patients with Alzheimer's disease: an examination of the first 10 years of CERAD data. J Clin Exp Neuropsychol. 1999;21:488-518.

14. Aharon-Peretz J, Israel O, Goldsher D, et al. Posterior cortical atrophy variants of Alzheimer's disease. Dement Geriatr Cogn Disord. 1999;10:483-487.

15. Johnson JK, Head E, Kim R, et al. Clinical and pathological evidence for a frontal variant of Alzheimer disease. Arch Neurol. 1999;56:1233-1239.

16. Lambon Ralph MA, Patterson K, Graham N, et al. Homogeneity and heterogeneity in mild cognitive impairment and Alzheimer's disease: a cross-sectional and longitudinal study of 55 cases. Brain. 2003;126(Pt 11):2350-2362.

17. Binetti G, Magni E, Padovani A, et al. Executive dysfunction in early Alzheimer's disease. J Neurol Neurosurg Psychiatry. 1996; 60:91-93.

18. Cummings JL. Cognitive and behavioral heterogeneity in Alzheimer's disease: seeking the neurobiological basis. Neurobiol Aging. 2000;21:845-861.

19. Mann UM, Mohr E, Gearing M, et al. Heterogeneity in Alzheimer's disease: progression rate segregated by distinct neuropsychological and cerebral metabolic profiles. J Neurol Neurosurg Psychiatry. 1992;55:956-959.

20. Kanne SM, Balota DA, Storandt M, et al. Relating anatomy to function in Alzheimer's disease: neuropsychological profiles predict regional neuropathology 5 years later. Neurology. 1998;50: 979-985.

21. Coen RF, Maguire C, Swanwick GR, et al. Letter and category fluency in Alzheimer's disease: a prognostic indicator of progression? Dementia. 1996;7:246-250.

22. Stern Y, Gurland B, Tatemichi TK, et al. Influence of education and occupation on the incidence of Alzheimer's disease. JAMA. 1994; 271:1004-1010.

23. Scarmeas N, Levy G, Tang MX, et al. Influence of leisure activity on the incidence of Alzheimer's disease. Neurology. 2001;57:2236-2242.

24. Charlson ME, Pompei P, Ales KL, et al. A new method of classifying prognostic comorbidity in longitudinal studies: development and validation. J Chronic Dis. 1987;40:373-383.

25. Buschke H, Fuld PA. Evaluating storage, retention, and retrieval in disordered memory and learning. Neurology. 1974;24:1019-1025.

26. Benton AL. The Visual Retention Test. New York: The Psychological Corp; 1955.

27. Kaplan E, Goodglass H, Weintraub S. Boston Naming Test. Philadelphia, PA: Lea \& Febiger; 1983. 
28. Benton AL, Hamsher K, Sivan AB. Manual for the Multilingual Aphasia Examination. 3rd ed. Iowa City, Iowa: AJA Associates, Inc; 1994.

29. Goodglass H, Kaplan D. The Assessment of Aphasia and Related Disorders. 2nd ed. Philadelphia, PA: Lea \& Febiger; 1983.

30. Wechsler D. Wechsler Adult Intelligence Scale-Revised. New York: The Psychological Corp; 1981.

31. Mattis S. Mental Status Examination for Organic Mental Syndrome in the Elderly Patient. New York: Grune \& Stratton; 1976.

32. Rosen W. The Rosen Drawing Test. Bronx, NY: Veterans Administration Medical Center; 1981.

33. Stern Y, Andrews H, Pittman J, et al. Diagnosis of dementia in a heterogeneous population. Development of a neuropsychological paradigm-based diagnosis of dementia and quantified correction for the effects of education. Arch Neurol. 1992;49:453-460.

34. Association AP. Diagnostic and Statistical Manual of Mental Disorders. Washington, DC: American Psychiatric Press, Inc; 1987.

35. Monsch AU, Bondi MW, Butters N, et al. Comparisons of verbal fluency tasks in the detection of dementia of the Alzheimer type. Arch Neurol. 1992;49:1253-1258.

36. Bracco L, Gallato R, Grigoletto F, et al. Factors affecting course and survival in Alzheimer's disease. A 9-year longitudinal study. Arch Neurol. 1994;51:1213-1219.
37. Kitabayashi $\mathrm{Y}$, Ueda $\mathrm{H}$, Tsuchida $\mathrm{H}$, et al. Relationship between regional cerebral blood flow and verbal fluency in Alzheimer's disease. Psychiatry Clin Neurosci. 2001;55:459-463.

38. Rosser A, Hodges JR. Initial letter and semantic category fluency in Alzheimer's disease, Huntington's disease, and progressive supranuclear palsy. J Neurol Neurosurg Psychiatry. 1994;57:1389-1394.

39. Henry JD, Crawford JR, Phillips LH. Verbal fluency performance in dementia of the Alzheimer's type: a meta-analysis. Neuropsychologia. 2004;42:1212-1222.

40. Keilp JG, Gorlyn M, Alexander GE, et al. Cerbral blood flow patterns underlying the differential impairment in category vs letter fluency in Alzheimer's disease. Neuropsychologia. 1999;37: $1251-1261$.

41. Roberson ED, Hesse JH, Rose KD, et al. Frontotemporal dementia progresses to death faster than Alzheimer disease. Neurology. 2005; 65:719-725.

42. Chan D, Fox NC, Jenkins R, et al. Rates of global and regional cerebral atrophy in AD and frontotemporal dementia. Neurology. 2001;57:1756-1763.

43. Knopman DS, DeKosky ST, Cummings JL, et al. Practice parameter: diagnosis of dementia (an evidence-based review). Report of the Quality Standards Subcommittee of the American Academy of Neurology. Neurology. 2001;56:1143-1153. 